\title{
Lecturas críticas de las nuevas propuestas de economía alternativa: ¿Qué hay de nuevo en lo nuevo?
}

\author{
A critical reading of new alternative economic and business \\ models: Whats New in the New? \\ J. FÉLIX LOZANO, EMMANUEL RAUFFLET \& ELSA GONZÁLEZ-ESTEBAN \\ Universitat Politècnica de València-HeC-Montreal \& Universitat Jaume I
}

Lozano, J. Félix; Raufflet, Emmanuel \& González-Esteban, Elsa (2018). Lecturas críticas de las nuevas propuestas de economía alternativa: ¿Qué hay de nuevo en lo nuevo? Recerca. Revista de Pensament $i$ Anàlisi, 23, pp. 9-18.

El cuestionamiento del sistema capitalista de mercado tiene una larga trayectoria, pero en las últimas décadas se han incrementado las propuestas que pretenden ofrecer alternativas a un sistema de mercado que favorece a los más aventajados e incrementa la desigualdad (Piketty 2014; Stiglitz 2013). Estas propuestas han surgido desde la experiencia de que el modelo actual dominante de capitalismo consumista es insostenible medioambientalmente e injusto socialmente.

Ni las críticas ni las propuestas alternativas al sistema capitalista son nuevas. Desde el mismo surgimiento de la sociedad industrial y la expansión del capitalismo, la economía social y el cooperativismo (en sus diversas variantes) han pretendido superar dicho modelo de generación y distribución de riqueza con propuestas centradas en las personas y sus necesidades antes que en los recursos y el capital. Pero ha sido con la entrada del siglo XXI, y sobre todo a partir de la crisis financiera de 2008, cuando han ganado protagonismo nuevas y diversas propuestas alternativas (unas veces radicales y otras reformistas) que tiene como finalidad reorientar las metas del sistema económico y cambiar las asimétricas relaciones que se establecen en las empresas. Estas nuevas propuestas, en ocasiones bajo el rótulo de nuevos modelos económicos o empresariales, están "presididos por esa vieja utopía de preservar y mejorar la vida, acrecentar la libertad, fortalecer la igualdad, impulsar la fraternidad» (Moreno, 2014). Entre otras iniciativas que persiguen este fin cabe mencionar: Economía colaborativa o compartida, Economía del bien común, Emprendimiento e innovación social, Economía circular y Economía civil. 
Estas son algunas de las nuevas propuestas que han ganado eco en los últimos años. Algunas de ellas pretenden una transformación radical del paradigma liberal del capitalismo consumista, mientras que otras proponen aprovechar su propia dinámica para beneficio de los menos aventajados o simplemente corregir los efectos no deseados sobre ellos.

El objetivo de este número de la Revista Recerca de Investigació i Análisis es realizar una lectura crítica de tales propuestas tratando de hacer balance de las mismas en su nivel normativo, así como mostrar las barreras o dificultades que están teniendo para su desarrollo práctico (Cortina et al., 2008). Los artículos que presentamos a continuación creemos que responden bien a este objetivo y presentan diversas perspectivas sobre los nuevos paradigmas de economía alternativa. El monográfico ha pretendido un equilibrio entre los artículos de crítica teórica y los de análisis de experiencias consolidadas y de las cuales podemos extraer conclusiones relevantes para la construcción de un mundo más justo. Comenzamos por los artículos que se centran una reflexión crítica normativa de las propuestas más extendidas (Laín; Portales), seguimos por dos artículos (Boni et. Ali; y Calvo) que suponen una reflexión sobre las dinámicas del sistema y cómo cambiarlo; continuamos por tres artículos de propuestas: Economía del Bien Común (Sanchis-Palacio y Vanessa Campos-Climent), economía civil (Zamagni) y Valor Social Añadido (Donati). Finalizamos la temática del monográfico con un análisis de las experiencias de economía alternativa en la ciudad de Valladolid (Ruíz-Valdepeñas et.Al.).Y cerramos el número con un artículo sobre la racionalidad lúdica y con una entrevista al profesor Dean MacCannell referente mundial en ética del turismo.

El primer artículo que presentamos, «Comunidades, racionalidad y mercados: una crítica institucional a la defensa emancipadora de la economía colaborativa» escrito por Bru Laín (Universidad de Barcelona) responde directamente a una de las preguntas esenciales que ha motivado este monográfico: Los nuevos modelos de economía alternativa, ¿hasta qué punto son realmente «alternativos», «reformistas» o son una mera «reformulación o adaptación» del modelo capitalista dominante? El profesor Laín comienza por intentar clarificar el concepto de Economía Colaborativa y concluye que es un concepto más pragmático que analítico donde las prácticas van por delante de los conceptos y que una definición que incluya fenómenos tan dispares como Airbnb, Wikipedia o los buertos urbanos es realmente complicado a la vez que necesario.

La segunda parte del artículo comienza por diferenciar dos grandes perspectivas de la Economía Colaborativa: a) la de tipo convencional, que 
considera que este tipo de economía es una evolución dentro del sistema capitalista que sigue la lógica de la optimización de recursos; y b) la de tipo emancipador que considera la economía colaborativa como transformación democrática, participativa y cooperativa de la economía liberal tradicional que puede llevarnos a un nuevo régimen más igualitario, sostenible y justo. El punto más relevante, a nuestro parecer, es su rigurosa crítica sobre esta segunda visión.

A juicio de Laín, la visión emancipadora de la economía colaborativa cae en dos errores: reproduce el sesgo psicológico de la economía liberal, y minusvalora el papel de las instituciones políticas y sociales. Concluye nuestro autor con la afirmación de que el simple hecho de hacer la economía más colaborativa no significa que se consigan mayores niveles de emancipación social. Interesante y controvertida afirmación que invita a continuar con el diálogo crítico.

En segundo lugar presentamos el artículo: «Emprendimiento social ¿alternativa o continuidad al sistema neoliberal?» que continúa con el análisis críticos de los nuevos modelos alternativos y de algunos de los conceptos que han ganado protagonismo en las últimas décadas. El profesor Portales (Universidad de Monterrey) cuestiona hasta qué punto la idea de emprendimiento social es alternativa o continuidad al neoliberalismo dominante. Ya anticipamos que su conclusión nos parece sólida y bien fundada, aunque sea menos ilusionante de lo que desearíamos.

Para comenzar, el autor presenta los rasgos esenciales del neoliberalismo. Con el concepto «neoliberalismo» ocurre como con muchos otros conceptos de gran presencia en el discurso político y social actual: todo el mundo lo nombra, pero pocos lo podrían definir con precisión. La precisión esquemática - pero rigurosa - de qué se entiende por neoliberalismo y sus consecuencias más significativas es un primer paso inicial para un posterior análisis comparativo con el emprendimiento social.

El elemento central de este artículo es la diferencia entre el emprendimiento social desde economía social o solidaria, y el emprendimiento social entendido desde la economía capitalista inclusiva. Aún cuando ambos modelos pretenden solucionar problemas sociales de forma económicamente sostenible, las diferencias entre ellos son significativas tanto en su desarrollo, como en sus fundamentos axiológicos. Mientras que desde el enfoque de economía social el emprendimiento se concibe como una estrategia de desarrollo local con una fuerte identidad de clase y procesos democráticos; el enfoque de economía capitalista inclusiva se concibe como una respuesta de negocio innovadora a una necesidad social. Desde 
esta caracterización la respuesta a la pregunta de si el concepto de emprendimiento social es una alternativa al modelo neoliberal o una consecuencia del mismo, se puede responder que el primer enfoque - economía social - pretende ser alternativa; mientras que el segundo - capitalismo inclusivo - se considera continuidad.Y esta distinción no es baladí ni desde el punto de vista teórico ni práctico. La distinción entre estos dos modelos tiene implicaciones teóricas que afectan a su legitimidad y sus presupuestos axiológicos y epistemológicos. También tiene implicaciones prácticas en el desarrollo de empresas e instituciones concretas como acertadamente apunta el profesor Portales al presentar dos modelos de empresa de la realidad mexicana.

Entender las dinámicas sociales y tener una perspectiva general de las propuestas de innovación social colectiva es el objetivo del tercer artículo que conforma este monográfico. El objetivo del artículo «Innovación social transformadora. Propuestas desde la innovación social colectiva para el desarrollo humano", presentado por Alejandra Boni, Sergio Belda y Victoria Pellicer, investigadores del Instituto Ingenio (CSIC-UPV) es presentar cómo las dinámicas de innovación social colectiva pueden generar transformaciones en los sistemas dominantes. Aquí se presentan iniciativa consolidadas en dos sectores centrales de nuestra sociedad: el consumo de productos agroalimentarios y el de la energía.

Los autores caracterizan la innovación social colectiva (grassroots innovations) como iniciativas de trasformación social guiadas por un interés de satisfacer problemas sociales o necesidades humanas, generando formas de organización democráticas y participativas y cuyo objetivo es cambiar el estatus quo del régimen socio-técnico dominante. La fundamentación ética normativa de este enfoque lo encuentran en el enfoque de Desarrollo Humano que entiende que las sociedades deben trabajar para la expansión de las capacidades de las personas. Estos autores consideran que hay una gran coherencia entre los principios y objetivos del enfoque del Desarrollo Humano y la perspectiva de la innovación social colectiva.

A nuestro juicio una gran fortaleza de este artículo es presentar dos experiencias de innovación social colectiva con una trayectoria consolidada (más de 5 años) y de cierto éxito como ejemplo de dos estrategias de transformación en dos sectores claves de nuestra sociedad: los grupos de consumo agroecológico y la cooperativa de generación, distribución y comercialización de energía (Som energia). Ambas iniciativas comparten los valores centrales del Desarrollo Humano (sostenibilidad, participación, diversidad y equidad), pero utilizan estrategias diferentes para transformar 
modelos dominantes basados, según nuestro autores, en los principios del capitalismo individualista liberal.

Concluyen los autores que estas iniciativas de Innovación Social Colectiva, tienen un gran valor social más allá de su éxito funcional. El mero hecho de generar nuevas formas de participación y generación de conocimiento, el fomento de la agencia y el cuestionamiento de los paradigmas dominantes contribuyen al Desarrollo Humano Sostenible.

En cuarto lugar el artículo presentado por Sara Calvo (Middlesex University London) «Applying Strategic Niche Management to understand how universities contribute to the development of social innovation niches: the case of the Social Innovation Scientific Park in Colombia» también supone una reflexión sobre una práctica de transformación social desde el fomento de la innovación social desde el ámbito universitario. La autora parte de la teoría de Strategic niche Management (SNM) y la aplica al contexto colombiano. Este artículo comparte con el anterior la idea de que los nichos son espacios desde los que se puede generar un cambio en los paradigmas socio-técnicos dominantes. En el artículo se detalla la experiencia del parque de innovación social promovido por la universidad Uniminuto como un ejemplo del papel que la educación superior puede jugar en el desarrollo de la innovación social.

La evaluación de esta experiencia se realizó con metodología cualitativa y se centró en analizar el impacto de estos procesos en el desarrollo y fortalecimiento de tres dimensiones: las redes sociales, la expectativas y visiones; y los procesos de aprendizaje.

Las conclusiones de la autora es que la experiencia ha sido exitosa, especialmente en lo que tiene que ver con el fortalecimiento de los procesos de aprendizaje, y que la educación superior puede jugar un papel muy relevante en el fomento de la Innovación social en Colombia, pero que es necesario expandir y fortalecer las redes de colaboración entre Universidades.

El artículo que presentamos en quinto lugar ha sido elaborado por los profesores Joan R. Sanchis-Palacio y Vanessa Campos-Climent (Universitat de València) y se centra en la fundamentación teórica de la Economía del Bien Común (EBC) (Felberg Felber 2012; Felber y Hagelberg 2017). Su objetivo es identificar las características esenciales del modelo y presentar una fundamentación teórica ante la extendida crítica de la falta de solidez teórica de la Economía del Bien Común.

Parten nuestros autores de la afirmación de que el modelo de economía capitalista basada en el individualismo posesivo no es sostenible y que se 
hace necesario un nuevo modelo económico, que, sin abandonar el mercado, transforme sus reglas y cambie la competencia por la cooperación. Su propuesta es que la EBC es un enfoque global e integral que supera otras iniciativas como la Responsabilidad Social Empresarial, la Economía Circular, o la Economía Social, aun cuando reconocen y asumen parte importante de sus aportaciones.

Según Sanchis-Palacio y Campos-Climent, el fundamento teórico de la EBC se encuentra en la teoría de los Stakeholder presentada por Freeman y en la teoría del Valor Compartido presentada por Porter y Kramer. En concreto, la matriz de Bien Común, que es el instrumento de aplicación de la EBC a las empresas, se estructura en torno a los cinco grupos de Stakeholders propuestos por la Stakebolder's theory: proveedores, propietarios y socios financieros, trabajadores, empleados, clientes y otras empresas, y el entorno social. Por su parte la Teoría del Valor Compartido coincide con la EBC en el objetivo de transformar la empresa desde la búsqueda de los beneficios particulares al beneficio social compartido.

El sexto artículo de este monográfico es el presentado por el profesor de la Universidad de Bolonia, Stefano Zamagni. Su propuesta de economía civil en la época de la cuarta revolución industrial es una propuesta que va en la línea de la EBC presentada en el artículo anterior aunque, a nuestro juicio, con una fundamentación filosófica más sólida. Su propuesta, en coherencia con publicaciones anteriores (Zamagni 2012), de una reinterpretación y recuperación de la economía civil se hace desde dos perspectivas: la primera es realizando un repaso histórico-crítico del surgimiento de la economía de mercado capitalista y del proceso de «olvido» de la economía civil dominante hasta el siglo XIX; y la segunda es demostrando la necesidad (y oportunidad) de la economía civil en momentos de transformación digital de la economía.

En la primera parte del artículo el profesor Zamagni nos recuerda brevemente la historia y la pluralidad de formas del sistema de mercado e insiste en cómo el capitalismo, a partir del s. XVIII, fue reemplazando gradualmente la lógica del bien común con el afán de beneficio. La concepción del mercado capitalista, basada en una la filosofía utilitarista y en una concepción hiper-simplificada del ser humano y sus motivaciones, ha tenido entre sus nefastas consecuencias el absolutizar la eficiencia como el máximo criterio del mercado, considerar el interés como la única motivación de las personas, y entender el consumo opulento como autorrealización.

En la segunda parte del artículo reflexiona cómo la revolución de la tecnología digital está transformando - a su juicio de forma negativa - la 
formación y la investigación. Considera nuestro autor que la transformación digital está profundizando en la impersonalidad y autointerés como criterios esenciales del mercado y en la idea del bomo oeconomicus como concepción antropológica dominante. Esta concepción «ideológica» y pesimista es una profecía que se auto-cumple con consecuencias muy negativas para la vida en sociedad.

Concluye el profesor Zamagni proponiendo el principio de fraternidad y la idea de homo reciprocans como elementos centrales de una economía civil que supere las limitaciones de la economía capitalista de mercado y favorezca el florecimiento de la diversidad humana.

En séptimo lugar, el artículo del profesor Donati (Universidad de Bolonia) - «Capital social, relaciones sociales y bienes públicos ¿qué conexiones?» - presenta una clarificación sobre el concepto y la interacción entre el capital social y los bienes públicos, y lo que a nuestro juicio es más importante, un modelo de dinámica generadora de capital social. El valor social añadido (VSA) se presenta como un concepto que aúna el capital social y los bienes públicos. En su artículo comienza cuestionando la visión defendida por algunos sociólogos del capital social, quienes afirman que el capital social no tiene conexión con la red social, e incluso que las redes sociales de proximidad generan cerrazón y tienen un impacto negativo en el capital social y el espíritu cívico de los individuos. Su propuesta es la contraria: el capital social asociativo favorece el desarrollo de los bienes públicos. Para él, la relación entre capital social y bienes públicos es circular en el sentido de que el capital social (re-)genera bienes públicos, pero a la vez una sociedad con sólidos bienes públicos incrementa el capital social.

Y para capturar este proceso, Donati propone el modelo de valor social añadido como un proceso de desarrollo temporal en el que partiendo de unas condiciones de partida se genera un regeneración de los bienes relacionales. El VSA da una medida de la capacidad de la red para producir sociabilidad. Este valor social añadido es resultado de la interacción de cuatro dimensiones del valor: valor de cambio, valor de uso, valor relacional, y valor de dignidad. Si estas dimensiones interactúan de forma equilibrada se genera capital social, pero si dominan las dimensiones de valor de cambio y valor de uso, se reduce el bien público y se consume capital social.

En octavo lugar presentamos el artículo «Práctica Social, Economía alternativa y espacios de proximidad en la ciudad de Valladolid» elaborado por Henar Pascual Ruiz-Valdepeñas, Esther Gil Álvarez \& Juan Carlos Guerra 
Velasco (Universidad de Valladolid). Es un artículo diferente a los anteriores y que, a nuestro juicio, los completa con una perspectiva empírica. El artículo se centra en una detallado análisis de las iniciativas de economía alternativa que se desarrollan en la ciudad de Valladolid. Comienzan por hacer una identificación y clasificación de las prácticas alternativas organizándolas en cuatro tipos básicos: producción de bienes y servicios, intercambio, consumo colaborativo y finanzas alternativas. Todas comparten los principios de autonomía, reciprocidad y democracia, y colaboración, a la vez que comparten una fuerte vinculación con el entorno local cercano y una estructura en red.

Las 24 iniciativas estudiadas son diversas en sus ámbitos de actuación (huertos urbanos, redes de consumo, centros sociales, etc.), su estructura legal (asociaciones, organizaciones sin forma jurídica), su tamaño, y su perfil ideológico; pero todas comparten la aspiración a confrontar el sistema capitalista en mayor o menor medida y a ser un espacio de aprendizaje. Sin duda, es éste uno de los puntos centrales de todas las iniciativas: la aspiración a generar conocimiento y fomentar espacios de reflexión y formación entre las personas que participan y difundir nuevas maneras de hacer economía.

Tras el estudio cualitativo de estas iniciativas nuestros autores concluyen que éstas tienen un creciente eco en la sociedad y que su potencial transformador será mucho mayor en la medida en que se profundice en su relación con experiencias de otras ciudades y se integren en visiones más amplias.

El último artículo de este número de RECERCA, «Una crítica a la modernidad a partir de la «inutilidad» del juego. El imperativo metanoético de Sloterdijk y la razón lúdica»» escrito por Xavier Gimeno (Colegio Aula 3) y Francisco Javier López (Penn State University) no se enmarca directamente en la línea del monográfico, pero como viene siendo habitual, la revista ofrece espacios y pública artículos que por su interés y calidad merecen ver la luz aunque no se enmarquen en el tema central propuesto. Sí que comparte con los artículos anteriores su cuestionamiento de la racionalidad instrumental dominante en la modernidad, ante la cual presenta la propuesta de la racionalidad lúdica de Bernar Suits (1990), y más en detalle de la razón ascética desarrollada por Sloterdijk. En esencia de lo que se trata es, como afirman los autores, de ^equilibrar los pesos de las diferentes racionalidades» algo que, sin duda, también afectaría a los modos de organizar la economía que ha sido el objetivo de este número monográfico. 
Finalmente, en el apartado de experiencias de este número monográfico se puede leer la interesante entrevista a Dean MacCannell. El emérito profesor de la University of California in Davis responde a las preguntas de José Luis López González sobre la dimensión ética del turismo. Es este ciertamente un tema interesante y novedoso. El creciente fenómeno del turismo tiene importantes impactos económicos, sociales y culturales que pueden ser valorados desde el punto de vista ético en dos direcciones opuestas. Para algunos autores el turismo ha sido valorado como un fenómeno que ayuda a conocer y valorar culturas diversas, que fomenta la generosidad y que combate el racismo y la xenofobia. Para otros autores es el máximo exponente del capitalismo hedonista que impone el «You must enjoy». Esta dicotomía entre la industria del turismo que explota los recursos naturales y culturales, y el turismo que pretende integrarse en las comunidades locales y fortalecer su economía y cultura, es la dicotomía que enmarca la reflexión ética sobre el turismo. Una gran entrevista que invita a la lectura detenida de las obras del profesor MacCannell.

\section{REFERENCIAS}

Cortina, A., García-Marzá, D. \& Conill, J. [eds.] (2008). Public Reason and Applied Ethics: the Ways of Practical Reason in a Pluralist Society, London: Routledge.

Felber, C. (2012). Economía del bien común: un modelo económico que supera la dicotomía entre capitalismo y comunismo para maximizar el bienestar de nuestra sociedad, Barcelona: Deusto.

Felber, C. \& G. Hagelberg (2017). The economy of common good.A Workable, Transformative Ethics-Based Alternative. The Next System Project, Feb. 27.

Moreno, J.A. (2014). Semillas de economía alternativa: ¿construyendo otro mundo? Mediterráneo económico, 26, 291-307.

Piketty, T. (2014). Capital in the twenty-first century, New York: Belknap Press.

Stiglitz, J. (2013). The price of inequality, New York: Penguin books.

Suits, B. (1990). The grasshopper: games, life, and Utopia. Boston: D. R. Godine

Zamagni, S. (2012). Por una economía del bien común. Madrid: Ciudad Nueva. 